\title{
Mechanical qubit-light entanglers in hybrid nonlinear qubit optomechanics
}

\author{
Victor Montenegro, ${ }^{1,2, *}$ G. D. de Moraes Neto, ${ }^{1,2}$ and Sougato Bose ${ }^{2}$ \\ ${ }^{1}$ Institute of Fundamental and Frontier Sciences, University of Electronic Science and Technology of China, Chengdu 610051, China \\ ${ }^{2}$ Department of Physics and Astronomy, University College London, Gower Street, London WC1E 6BT, United Kingdom
}

(Received 24 June 2019; revised manuscript received 27 August 2019; published 8 November 2019)

\begin{abstract}
Interfacing between matter qubits and light is a crucial provision for numerous quantum technological applications. However, a generic qubit may not directly interact with a relevant optical field mode, and hence, one could necessitate adjusting frequencies to match resonance conditions between parties. In this work, we show how a parametric coupling of the qubit with a mechanical oscillator, in conjunction with the trilinear radiation pressure coupling of the same object with light, can induce maximal qubit-light entanglement at an optimal time. Furthermore, we show how our method enables conditional (dynamical) nonclassical state preparation of the optical field via qubit measurement in the weak (moderate-to-strong) optomechanical coupling regime. Our scheme benefits from not requiring any cooling of the mechanical component and not needing an adjusting of the detunings and transition frequencies to have resonance between any pairs of quantum systems.
\end{abstract}

DOI: 10.1103/PhysRevA.100.052310

\section{INTRODUCTION}

Recent works on multipartite hybrid quantum systems attest to be a promising avenue to control and manipulate quantum systems of different nature [1-3]. Mostly, such hybrid platforms serve as multitasking modular architectures; hence, each building block (commonly with different frequencies) of the assembled unit plays a different role in encoding, processing, distributing, and reading out the quantum information. One appropriate example to observe the benefits of hybrid schemes is to consider nodes allocated in a quantum network (for light-mediated qubit-oscillator systems we refer the interested reader to Refs. [2,4], for example). There, the link between distant nodes is via photonic qubits (or propagating phonons in extended phonon waveguides [5]), while the encoding (or storing) can employ the node itself, utilizing matter qubits or mechanical oscillators for this purpose [6].

On the other hand, the interplay between matter-light parties typically demands resonant (or quasiresonant) interactions between a qubit and a cavity field [6] - this is the case, for example, for trapped single or collective two-level atoms inside a cavity $[2,7]$. The qubit-cavity direct coupling could be used to either map the qubit state to a cavity field so that it was carried and fed into a distant cavity via the light [8-10], or to entangle a qubit maximally with the field in a cavity. Subsequent joint detections of the light fields from two separate cavities could be used to entangle the qubits maximally in a heralded manner [11].

In recent years a plethora of other qubits has surfaced which have frequencies in the microwave and radio-frequency range [12-16]. For these, an alternative strategy has been suggested where the qubit and the optics interact with a mechanical mediator, i.e., a hybrid quantum scheme with no direct coupling between the qubit and the cavity field. These systems

\footnotetext{
*vmontenegro@uestc.edu.cn
}

have shown the successful linking of distant qubits through optomechanics [5,17-19]. However, these schemes rely on an exchange of excitations between systems: Jaynes-Cummings (beam-splitter) interaction between the qubit and the mechanics (mechanics and light). In the case of Jaynes-Cummings combined with beam-splitter interaction, it is intuitive that the former and latter Hamiltonians swap quantum states from the qubit to mechanics and mechanics to light, respectively [1]. Consequently, schemes relying on the exchange of excitations are only ensured at the cost of an appropriate adjustment of detunings of the fields, hence driving the qubit and the optical field from their respective transitions by precisely the mechanical frequency.

In this work, we wonder whether we can relax the above resonance conditions between parties and thus to combine distinct elements into an off-resonant hybrid system. In other words: is it possible to entangle two nonresonant and noninteracting (directly) qubit-light subsystems mediated by a mechanical object? To address this question, we make use of parametric Hamiltonians - which are not of a state swapping type by nature. It is, therefore, relevant to explore whether parametric interactions of qubits with mechanics and the parametric trilinear optomechanical interaction can be fruitfully used to entangle a qubit with light.

Specifically, we study a system, as shown in Fig. 1, where a generic qubit becomes entangled to a cavity mode mediated through a mechanical object. Here we consider the qubit directly coupled to the mechanical oscillator position. Such qubit-oscillator Hamiltonians are synthesized in a variety of ways, notably through magnetic field gradients [20] or through capacitive couplings [21]. Additionally, for the oscillator-cavity subsystem, we exploit the nonlinear radiation pressure interaction. Very recently, related hybrid qubitoptomechanics systems are of interest fundamentally in giving rise to attractive polaritonic states involving mechanics [7] and tripartite entanglement [22]. Through these Hamiltonians, the most sensible task perhaps is to look for entanglement 


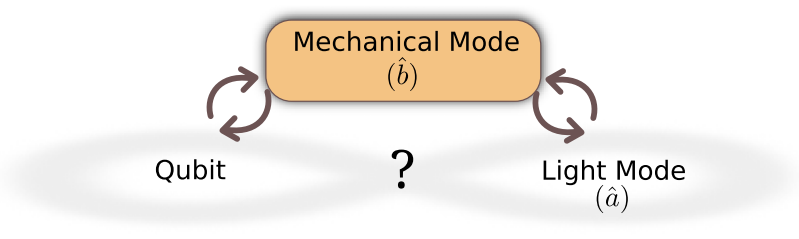

FIG. 1. A qubit interacts with a mechanical oscillator [with boson operator $(\hat{b})]$, while the latter object couples to a quantized light field $(\hat{a})$. There is no direct qubit-light interaction but that which is mediated through the mechanical subsystem. We wonder in this hybrid tripartite quantum system whether one could reach, dynamically and under Hamiltonian parametric interactions, a high degree of qubit-light quantum entanglement.

between the qubit and the optical fields. Even for this task, whether the entanglement will be "transitive" in nature is a priori unclear. We show here that it is indeed possible to achieve indirect oscillator-mediated qubit-cavity entanglement under parametric Hamiltonians without the necessity for adjusting resonances.

The rest of the article reads as follows: In Sec. II, we focus our attention on the entanglement dynamics for three relevant initial states. For the initial pure states considered by us, we give a closed form for the entanglement dynamics [23], which allows us to tune the interaction couplings to reach maximal qubit-cavity entanglement at a specific time. In Sec. III we devote the qubit-light entanglement analysis in the presence of decoherence. For this study, we solved the master equation [24] in a dressed picture, as the single-photon radiation strength operates in the moderate-to-strong optomechanical regime. In Sec. IV, we investigate the conditional preparation of nonclassical states of the optical field by (i) its dynamics alone and (ii) via measurements on the qubit subsystem. Finally, in Sec. V, we conclude and outline the main results of our work.

\section{TRIPARTITE HYBRID DYNAMICS}

We study a hybrid tripartite qubit-optomechanical system as sketched in Fig. 1. In particular, we consider a generic qubit coupled dispersively (and nonresonantly) to a mechanical object, while a single cavity mode interacts with the latter one via trilinear radiation pressure interaction. The Hamiltonian in the interaction picture of the free energy of the light field and the qubit is $(\hbar=1)$ :

$$
\hat{H}_{\text {int }}=\omega_{m} \hat{b}^{\dagger} \hat{b}-\left(g_{0} \hat{a}^{\dagger} \hat{a}+\lambda_{0} \hat{\sigma}_{z}\right)\left(\hat{b}+\hat{b}^{\dagger}\right),
$$

where $\omega_{m}$ stands for the mechanical frequency, $g_{0}$ is the cavity-oscillator radiation pressure coupling, and $\lambda_{0}$ is the qubit-oscillator coupling strength; $\hat{a}(\hat{b})$ is the boson operator for the cavity (oscillator), respectively, and $\hat{\sigma}_{z}$ is the Pauli $z$ matrix for the qubit. Here, we would like to stress that, from Eq. (1), one may notice that the individual Hamiltonians do indeed entangle a qubit-mechanical oscillator pair and an optical field-mechanical oscillator pair, but whether also a qubit-optical field entanglement will result from this is hard to guess.

To solve the quantum dynamics, we proceed to derive the evolution operator for the Hamiltonian in Eq. (1) as done previously in Refs. [25-27]:

$$
\hat{U}(t)=e^{i\left(g \hat{a}^{\dagger} \hat{a}+\lambda \hat{\sigma}_{z}\right)^{2}(t-\sin t)} e^{\left(g \hat{a}^{\dagger} \hat{a}+\lambda \hat{\sigma}_{z}\right)\left(\eta \hat{b}^{\dagger}-\eta^{*} \hat{b}\right)} e^{-i t \hat{b}^{\dagger} \hat{b}},
$$

where $\eta \equiv \eta(t)=1-e^{-i t}$, and $\left\{g=g_{0} / \omega_{m}, \lambda=\lambda_{0} / \omega_{m}\right\}$ are the scaled coupling parameters.

Motivated for the indirect generation of qubit-cavity quantum entanglement, a simple inspection of Eq. (2) suggests some directions regarding the initial states for the qubit and the cavity field. Those indications are due to the dispersive interaction, i.e., a Hamiltonian conserving both the qubit and optical excitations. Hence, to attempt qubit-light entanglement generation, one is required to initialize the qubit (cavity) different from an eigenstate of $\hat{\sigma}_{z}$ (Fock number state). Otherwise, no entanglement between the qubit (or the cavity) can be generated with the rest of the subsystems, as they persist in disentangling during the evolution. For the sake of simplicity, we will consider throughout this work an initial qubit superposition $1 / \sqrt{2}(|\uparrow\rangle+|\downarrow\rangle)$, where some relevant initial states for the cavity state and the mechanical object will be studied.

\section{A. Optical qubit and mechanical oscillator in coherent state}

Let us evolve the system from an initial superposition of Fock number states $1 / \sqrt{2}(|0\rangle-|1\rangle)$ for the cavity field [28] (optical qubit). This state finds fertile ground for quantum information applications. Although easy to formulate mathematically, this state remains difficult to prepare experimentally. Preparation techniques of the optical mode in a state $\sim|0\rangle-|n\rangle$ have been reported previously [29-32], $n=1$ being the most simple state to generate in the laboratory.

On the other hand, we initialize the mechanical object in a coherent state with an amplitude of $\beta>0$. With these initial conditions, we intend to unravel the tripartite dynamics from states with low Hilbert spaces. By evoking the unitary operator from Eq. (2), we obtain the following wave function:

$$
\begin{aligned}
|\psi(t)\rangle= & \frac{1}{2}\left[e^{i \lambda^{2}(t-\sin t)} e^{i \lambda \beta \sin t}|\uparrow\rangle|0\rangle\left|\beta e^{-i t}+\lambda \eta\right\rangle\right. \\
& -e^{i(g+\lambda)^{2}(t-\sin t)} e^{i(g+\lambda) \beta \sin t}|\uparrow\rangle|1\rangle\left|\beta e^{-i t}+(g+\lambda) \eta\right\rangle \\
& +e^{i \lambda^{2}(t-\sin t)} e^{-i \lambda \beta \sin t}|\downarrow\rangle|0\rangle\left|\beta e^{-i t}-\lambda \eta\right\rangle \\
& -e^{i(g-\lambda)^{2}(t-\sin t)} e^{i(g-\lambda) \beta \sin t}|\downarrow\rangle|1\rangle \mid \beta e^{-i t} \\
& +(g-\lambda) \eta\rangle] .
\end{aligned}
$$

To investigate the quantum correlations of the qubitoptomechanical wave function in Eq. (3), we proceed to calculate the quantum entanglement between bipartite systems. In general, throughout this work, the entanglement will be mainly computed using the negativity $[\mathcal{N}(t)][33]$, a quantity defined as

$$
2 \mathcal{N}(t)=\sum\left|\varepsilon_{i}\right|-\varepsilon_{i}
$$

where $\varepsilon_{i}$ are the eigenvalues of the partially transposed reduced density matrix at fixed time $t$.

In Fig. 2 we illustrate the dynamics of quantum entanglement for a set of qubit-optomechanical coupling values $\{g, \lambda\}$. Concretely, we have computed the qubit-cavity $\left[\mathcal{N}(t)_{q, c}\right]$, the qubit-oscillator $\left[\mathcal{N}(t)_{q, o}\right]$, and the oscillator-cavity negativity $\left[\mathcal{N}(t)_{c, o}\right]$. In Fig. 2(a), we chose $g=0.1$ and $\lambda=0.4$ 

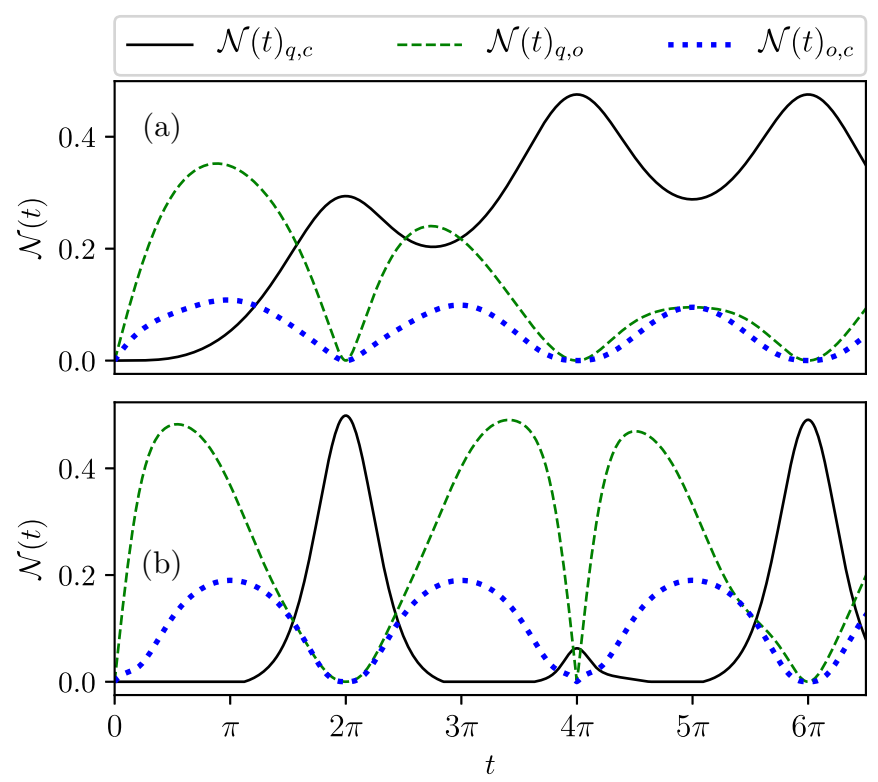

FIG. 2. Negativity dynamics $[\mathcal{N}(t)]$ for different reduced density matrices. We plot the qubit-cavity $\left[\mathcal{N}(t)_{q, c}\right.$, solid line], the qubitoscillator $\left[\mathcal{N}(t)_{q, o}\right.$, dashed line], and the oscillator-cavity negativity $\left[\mathcal{N}(t)_{o, c}\right.$, dotted line $]$ for different qubit-optomechanical coupling values. Top (bottom) panel considers $g=0.2$ and $\lambda=0.25$ ( $g=0.2$ and $\lambda=0.625) ; \beta=1$.

(for $\beta=1$ ). Notice that the mechanical oscillator disentangles from the rest of the subsystems at each cycle, i.e., at times $2 \pi / \omega_{m} \times l$ ( $l$ being an integer), the qubit-oscillator (and cavity-oscillator) negativity vanishes to zero-this can be seen as $\eta(t=2 \pi)=0$, and therefore, $\left|\beta e^{-2 \pi i} \pm \lambda \eta\right\rangle=$ $\left|\beta e^{-2 \pi i}+(g \pm \lambda) \eta\right\rangle=|\beta\rangle$. This is crucial. The qubit-light entanglement at multiples of $2 \pi$ is independent of the coherentstate preparation for the mechanical oscillator, reaching its first maximum of $\mathcal{N}(t)_{q, c}=0.5$ at $t=4 \pi$. Thus, the initially disentangled qubit-cavity subsystems have been indirectly (and maximally) entangled through a mechanical object.

Furthermore, one may require possession of highly entangled states at the earliest in the quantum dynamics, for instance, to avoid detrimental effects due to decoherence or for quantum computing/processing purposes. To reach the maximum qubit-cavity negativity at $t=2 \pi$, we need to optimize the set of qubit-optomechanical parameters $\{g, \lambda\}$ in the negativity function. However, albeit quite manageable to compute numerically, a closed analytical form is usually challenging to obtain. To overcome this obstacle, we proceed to derivate a simple form encompassing several partitions of the tripartite system. This simple, yet rich procedure [23] shows that an appropriate addition (subtraction) of individual entropies should be capable of quantifying the degree of entanglement within each of the subsystems. The intrinsic qubit-cavity entanglement is defined as follows:

$$
\mathcal{E}_{q, c}(t)=\mathcal{S}(t)_{q}+\mathcal{S}(t)_{c}-\mathcal{S}(t)_{o}
$$

In the above, $\mathcal{S}(t)_{i}=1-\operatorname{Tr}\left[\hat{\rho}_{i}^{2}(t)\right]$ is the linear entropy, and $\hat{\rho}_{i}(t)=\operatorname{Tr}_{j, k} \hat{\rho}_{i, j, k}$ is the corresponding reduced density matrix. With the above definition, the intrinsic qubit-cavity entanglement reduces to

$$
\begin{aligned}
\mathcal{E}_{q, c}(t)= & \frac{1}{8}\left(e^{2(g+2 \lambda)^{2}(\cos t-1)}+e^{2(g-2 \lambda)^{2}(\cos t-1)}+2\right. \\
& \left.-2\left[e^{2 g^{2}(\cos t-1)}+e^{8 \lambda^{2}(\cos t-1)}\right] \cos [4 g \lambda(t-\sin t)]\right)
\end{aligned}
$$

which, for the special case of $t=2 \pi$, simplifies to

$$
\mathcal{E}_{q, c}(t=2 \pi)=\sin ^{2}(4 g \lambda \pi)
$$

From the above equation, we can readily notice that $\mathcal{E}_{q, c}(t=2 \pi)$ reaches its first maximum at any coupling combinations given by $g \lambda=1 / 8$. In Fig. 2(b) we model this case, where we have considered $g=1 / 5=0.2$ and $\lambda=$ $5 / 8=0.625$. Any tuple $g \lambda=1 / 8$ would deliver maximal entanglement. Nevertheless, as $g$ decreases to the weak-tomoderate radiation pressure regime, one needs $\lambda$ to increase to the strong spin-mechanical regime. On this matter, notice that both values $\{g, \lambda\}=\{0.2,0.625\}$ are within the current experimental feasibility. Vast efforts to increase the singlephoton radiation pressure coupling have been pursued during the last years. For example, the above moderate optomechanical operational regime of $g=g_{0} / \omega_{m} \lesssim 0.2$ has been reported in quantum cavity pulsed optomechanics [34] and other setups [35-39].

As seen from this section, we have fully accomplished the indirect qubit-cavity entanglement mediated through a mechanical object. However, even though an initial optical qubit gives us insight into the qubit-optomechanical evolution and the entanglement dynamics, this particular preparation for the light field is hard to access experimentally. For that reason, in the next section we will study the merits and demerits of a more feasible preparation for the optics: a coherent state.

\section{B. Coherent states for the light and the mechanical oscillator}

Assume that initially both the mechanics and the optics fields are in a coherent-state preparation, $|\beta\rangle$ and $|\alpha\rangle$, respectively. On the one hand, to generate such a state for the light, one can notice that once the cavity has no intracavity photons, we can drive the vacuum state towards a displaced vacuum state $|\alpha\rangle$ by using an external laser. This process acts on a timescale which is much shorter than the timescale of the oscillator's motion and hence does not have a significant perturbation in the mechanical dynamics - a similar strategy generates a coherent state for the mechanical oscillator. Several theoretical and experimental techniques have been proposed to reach the ground-state cooling for mechanical oscillators and even recently demonstrated cooling of mechanical vibrational modes close to the quantum ground state [40].

The initial quantum state $|\psi(0)\rangle=1 / \sqrt{2}(|\uparrow\rangle+|\downarrow\rangle)|\alpha\rangle|\beta\rangle$ then evolves according to

$$
|\psi(t)\rangle=\sum_{n=0}^{\infty}\left(C_{n}^{+}(t)|\uparrow\rangle\left|\phi_{n}^{+}(t)\right\rangle+C_{n}^{-}(t)|\downarrow\rangle\left|\phi_{n}^{-}(t)\right\rangle\right)|n\rangle,
$$

where

$$
C_{n}^{ \pm}(t)=\frac{\alpha^{n}}{\sqrt{2 n !}} e^{-|\alpha|^{2} / 2} e^{i(g n \pm \lambda)^{2}(t-\sin t)} e^{i(g n \pm \lambda) \operatorname{Im}(\eta \beta)},
$$


and $\left|\phi_{n}^{ \pm}(t)\right\rangle=\left|\beta e^{-i t}+(g n \pm \lambda) \eta\right\rangle$ is a coherent state for the mechanical oscillator.

In contrast to our previous section, where the optics were restricted to a two-level system-being zero and one photon, here the explicit optomechanical Kerr-like term $e^{i(g n \pm \lambda)^{2}(t-\sin t)}$ in $C_{n}^{ \pm}(t)$ plays a significant role in the dynamics. We expect that with the aid of the explicit number of photons $n$ in the Kerr phase, we can reach the maximum entanglement for weaker values of $g$. On the other hand, each qubit eigenstate dynamically couples to different coherent amplitudes $\left|\phi_{n}^{ \pm}(t)\right\rangle$, as each qubit component effectively shifts the mechanical potential proportional to $\lambda_{0}-$ a shift also found in the previous section. Moreover, the optomechanical interaction from coherent amplitudes indicates a dynamic squeezing of the mechanical quadratures of the reduced density matrix of the mechanical oscillator.

To further explore the bipartite dynamics, we proceed to derive the reduced density matrices as follows:

$$
\begin{aligned}
&\left.\hat{\rho}_{o, c}(t)=\sum_{\substack{n, m=0 \\
j=\{+,-\}}}^{\infty} C_{n}^{j}(t) C_{m}^{j *}(t) \mid n, \phi_{n}^{j}\right)\left\langle m, \phi_{m}^{j}\right|, \\
& \hat{\rho}_{q, o}(t)=\sum_{n=0}^{\infty} C_{n}^{+}(t) C_{n}^{+*}(t)\left|\uparrow, \phi_{n}^{+}\right\rangle\left\langle\uparrow, \phi_{n}^{+}\right|+C_{n}^{+}(t) \\
& \times C_{n}^{-*}(t)\left|\uparrow, \phi_{n}^{+}\right\rangle\left\langle\downarrow, \phi_{n}^{-}\left|+C_{n}^{-}(t) C_{n}^{+*}(t)\right| \downarrow, \phi_{n}^{-}\right\rangle \\
& \times\left\langle\uparrow, \phi_{n}^{+}\left|+C_{n}^{-}(t) C_{n}^{-*}(t)\right| \downarrow, \phi_{n}^{-}\right\rangle\left\langle\downarrow, \phi_{n}^{-}\right|,
\end{aligned}
$$

being the oscillator-cavity $(o, c)$ and the qubit-oscillator $(q, o)$ bipartite subsystems, respectively. And

$$
\begin{aligned}
\hat{\rho}_{q, c}(t)= & \sum_{n, m=0}^{\infty}\left(C_{n}^{+}(t) C_{m}^{+*}(t) \phi_{m n}^{++}|\uparrow\rangle\langle\uparrow|+C_{n}^{+}(t)\right. \\
& \times C_{m}^{-*}(t) \phi_{m n}^{-+}|\uparrow\rangle\left\langle\downarrow\left|+C_{n}^{-}(t) C_{m}^{+*}(t) \phi_{m n}^{+-}\right| \downarrow\right\rangle\langle\uparrow| \\
& \left.+C_{n}^{-}(t) C_{m}^{-*}(t) \phi_{m n}^{--}|\downarrow\rangle\langle\downarrow|\right) \otimes|n\rangle\langle m|
\end{aligned}
$$

stands for the qubit-cavity $(q, c)$ reduced system; we defined $\phi_{m n}^{i j}=\left\langle\phi_{m}^{i} \mid \phi_{n}^{j}\right\rangle$ as the inner product between coherent states.

In analogy with the previous section, it is straightforward to notice that for each cycle of the mechanical object $(t=2 \pi \rightarrow$ $\eta=0$ ), the oscillator disentangles from the cavity as well as the qubit state. It is worth expressing the wave function at such particular times:

$$
\begin{aligned}
|\psi(t=2 \pi)\rangle_{q, c}= & e^{-|\alpha|^{2} / 2} \sum_{n=0}^{\infty} \frac{\alpha^{n}}{\sqrt{2 n !}}\left[e^{i(g n+\lambda)^{2} 2 \pi}|\uparrow\rangle\right. \\
& \left.+e^{i(g n-\lambda)^{2} 2 \pi}|\downarrow\rangle\right]|n\rangle .
\end{aligned}
$$

We investigate the entanglement dynamics of each bipartite system in Fig. 3. When compared to the previous case, two clear advantages arise: (i) the qubit-cavity entanglement reaches its maximum faster than in the optical qubit scenario, and (ii) the qubit-cavity entanglement does not heavily decrease (nor drop to zero) for a larger time window. Other values are $\beta=2, \alpha=2, g=0.2, \lambda=0.25$.

To find a suitable set of qubit-optomechanical couplings such as would maximize the qubit-cavity entanglement, we follow the same procedure as in the previous section. The

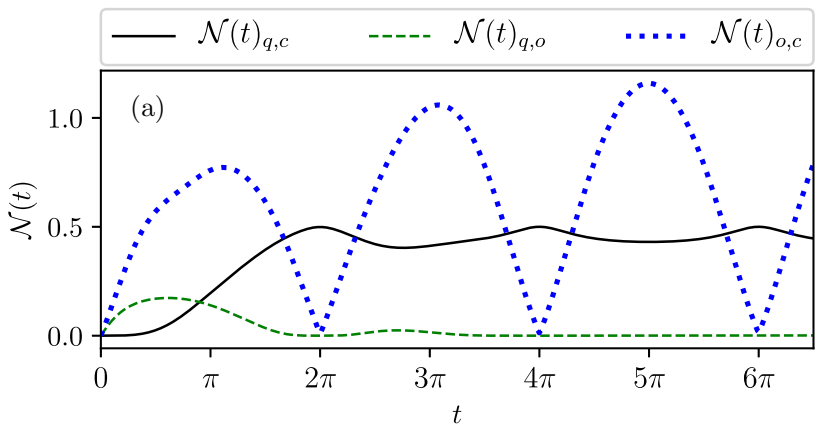

(b) Coherent light field

(c) Superposition light field

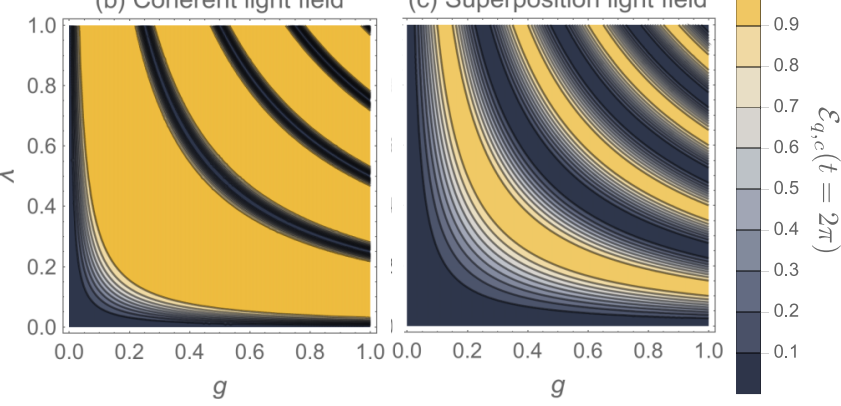

FIG. 3. (a) Different bipartite entanglement dynamics for an initial cavity and mechanical oscillator in coherent states. In (b) and (c) we plot the intrinsic entanglement at $t=2 \pi$ for the current optics in the coherent case [see Eq. (14)] and the previous optical qubit scenario [see Eq. (7)], respectively. Other values are $\beta=2, \alpha=$ $2, g=0.2, \lambda=0.25$.

linear entropies can be obtained directly from the reduced density matrices already expressed in Eqs. (10)-(12). The intrinsic qubit-cavity entanglement at $t=2 \pi$ for the coherent case reads as

$$
\begin{aligned}
\mathcal{E}_{q, c}(t=2 \pi) & =1-\sum_{\substack{n=0 \\
m=0}}^{\infty} \frac{e^{-2 \alpha^{2}} \alpha^{2(m+n)} \cos [8 \pi g \lambda(m-n)]}{m ! n !} \\
& =1-e^{-4 \alpha^{2} \sin ^{2}[4 g \lambda \pi]}
\end{aligned}
$$

Notice from the above Eq. (14) that $\sin (4 g \lambda \pi)$ appears as in the previous section. However, while for an initial optical qubit the condition $4 g \lambda \pi=\pi / 2$ gives rise directly to maximal entanglement, for the coherent case, the same condition drives asymptotically the intrinsic entanglement towards unity. Moreover, as a direct consequence from the optomechanical evolution in the presence of coherent light, we stress that the number of photons $\left[\alpha^{2}\right.$ in Eq. (14)] also assists the maximal value of the entanglement, as we previously suggested. We compare both situations in the bottom panels of Fig. 3.

We have shown that to accomplish a high indirect qubitcavity entanglement one needs to operate in the regime when both $\{g, \lambda\}$ are comparable. Within these values, we can reach a higher qubit-cavity quantum entanglement at faster mechanical cycles in the dynamics. Let us now comment on the entanglement dynamics for two relevant operational regimes.

First, let us consider the case when the single-photon radiation pressure coupling dominates over the spin-mechanical 
coupling, i.e., scaled frequencies $g \gg \lambda$. In this regime, where the optomechanical coupling dominates, one can observe (for a large number of mechanical cycles $t \gg 1$ ) that the tripartite system evolves mainly as a coherent evolution between the light and the mechanics. In the opposite case, when the spin-mechanics interaction dominates, $g \ll \lambda$, we find that the qubit entangles the mechanical object. In both cases, it is also possible to achieve coherent dynamics between the qubit-cavity systems; however, high entanglement between those are generated only at $t \gg 1$.

\section{Coherent state for the cavity field and a thermalized mechanical oscillator}

A step forward to study the bipartite entanglement under different initial preparations is to consider an initial coherent state for the light field and a mechanical oscillator at temperature $T$. In principle, as we intend to utilize the mechanical object solely as a mediator, it is more precise to consider an initial thermal state representation for the mechanical subsystem. Thus, let us consider in this section a cavity state prepared in a coherent state, while the mechanical object is in a thermal state at temperature $T$ :

$$
\hat{\rho}_{\mathrm{th}}(0)=\frac{1}{\pi \bar{n}} \int|\beta\rangle\langle\beta| e^{-\frac{|\beta|^{2}}{\bar{n}}} d^{2} \beta,
$$

where $\bar{n}=\left[\exp \left(\hbar \omega_{m} / k_{B} T\right)-1\right]^{-1}$ stands for the thermal occupancy number and $k_{B}$ is the Boltzmann constant. By solving the Schrödinger equation, we can readily get the tripartite normalized density matrix as follows:

$$
\begin{aligned}
\hat{\rho}(t)= & \sum_{n, m=0}^{\infty}|n\rangle\langle m| \otimes\left[|\uparrow\rangle\left\langle\uparrow\left|\otimes \hat{\rho}_{++}^{t h}+\right| \downarrow\right\rangle\langle\downarrow| \otimes \hat{\rho}_{--}^{t h}\right. \\
& \left.+|\uparrow\rangle\left\langle\downarrow\left|\otimes \hat{\rho}_{+-}^{t h}+\right| \downarrow\right\rangle\langle\uparrow| \otimes \hat{\rho}_{-+}^{t h}\right],
\end{aligned}
$$

where we have simplified the notation as $\hat{\rho}_{a b}^{t h} \equiv \hat{\rho}_{a b}^{t h}(n, m, t)=$ $1 /(\pi \bar{n}) \times \int C_{n}^{a}(t) C_{m}^{* b}(t)\left|\phi_{a}^{n}(t)\right\rangle\left\langle\phi_{b}^{m}(t)\right| e^{-|\beta|^{2} / \bar{n}} d^{2} \beta, \quad$ where $\{a, b\}$ might be + or - .

To observe the impact of the temperature of the mechanical oscillator in the qubit-cavity dynamics, we proceed to compute the reduced density matrix between the qubit and the cavity. Tracing out the mechanical degrees of freedom leads to an analytical result of the qubit-cavity subsystems:

$$
\begin{aligned}
\hat{\rho}(t)_{q, c}= & \sum_{n, m=0}^{\infty}|n\rangle\langle m| \otimes\left[|\uparrow\rangle\left\langle\uparrow\left|I_{++}+\right| \downarrow\right\rangle\langle\downarrow| I_{--}\right. \\
& \left.+|\uparrow\rangle\left\langle\downarrow\left|I_{+-}+\right| \downarrow\right\rangle\langle\uparrow| I_{-+}\right] .
\end{aligned}
$$

In the above, the terms

$$
I_{a b}=\frac{1}{\pi \bar{n}} \int C_{n}^{a}(t) C_{m}^{* b}(t)\left\langle\phi_{b}^{m}(t) \mid \phi_{a}^{n}(t)\right\rangle e^{-\frac{|\beta|^{2}}{\bar{n}}} d^{2} \beta,
$$

where $\{a, b\}$ might be + or -, are Gaussian integrals. Thus, it is convenient to compute the integral considering Cartesian coordinates $d^{2} \beta=d \operatorname{Re}[\beta] d \operatorname{Im}[\beta]$.

In Fig. 4 , we present the qubit-cavity entanglement in the presence of several phonon numbers on average $(\bar{n})$. From the figure, we readily notice two features: (i) the qubit-cavity entanglement suffers a decrement when in the presence of higher

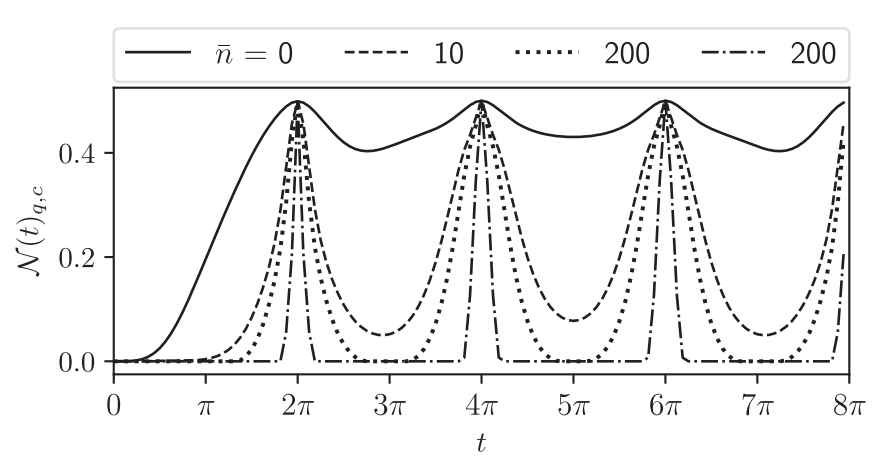

FIG. 4. Qubit-cavity entanglement dynamics $\mathcal{N}(t)_{q, c}$ mediated by a thermalized mechanical oscillator with $\bar{n}$ phonons on average.

phonon occupancy number, with an important reduction of entanglement specifically in regions where $t$ is a multiple of $\pi$. This dynamics can be understood as the mechanical oscillator remains mostly static as $\bar{n}$ increases, i.e., both $g$ and $\lambda$ have a little effect on the mechanical oscillator between cycles. Furthermore, notice that for megahertz mechanical oscillators at milliKelvin temperatures, the phonon occupancy is $\bar{n} \sim$ 10 , meaning that for current experimental technologies one can expect to have qubit-cavity entanglement above zero for several mechanical cycles. (ii) The qubit-cavity entanglement is independent of $\bar{n}$ at each $t$ multiples of $2 \pi$. This fact is a consequence of the complete disentanglement of the mechanical object from both parties.

To prove the second feature, we can notice from Eq. (16) that the mechanical object disentangles at each mechanical cycle. Nonetheless, when compared to previous cases where the tripartite state remains pure throughout the dynamics, here it is not a direct consequence. The qubit-cavity reduced bipartite undergoes a change from the initially mixed state to a pure state at each oscillator cycle. Moreover, it coincides with the previous wave function derived before [see Eq. (13)], being independent also from the thermal occupancy number $\bar{n}$ :

$$
\begin{aligned}
|\psi(t=2 \pi)\rangle_{q, c}= & e^{-|\alpha|^{2} / 2} \sum_{n=0}^{\infty} \frac{\alpha^{n}}{\sqrt{2 n !}}\left[e^{i(g n+\lambda)^{2} 2 \pi}|\uparrow\rangle\right. \\
& \left.+e^{i(g n-\lambda)^{2} 2 \pi}|\downarrow\rangle\right]|n\rangle .
\end{aligned}
$$

Despite the coinciding wave functions in Eqs. (13) and (19), the scenario involving an evolved thermal mechanical oscillator diverges from the coherent state in the transient intervals. For instance, for a time interval $2 \pi<t<10 \pi$ and $\bar{n}=|\beta|^{2}=4$, the oscillator always becomes disentangled at time multiples of $t=2 \pi$. However, quantum entanglement oscillating near its maximum value [as in Fig. 3(a)] was not reported for any set of qubit-optomechanical coupling strengths in the domain $0<\{g, \lambda\} \leqslant 1$ in the case of an initial thermal mechanical oscillator.

\section{DYNAMICS IN THE PRESENCE OF LOSSES}

Quantum correlations, or ultimately, quantum coherence, generally suffer from a decrement when the relevant system of interest evolves in contact with their surroundings. In the absence of an engineered reservoir or suitable dissipative 
mechanism, quantum correlations are a frail resource in the presence of noise. In this section, we will study the effects of the impact of energy losses on the quantum dynamics, mainly centering our attention on the qubit-cavity subsystems. The resulting detrimental effects on the unitary evolution stems from several decoherence channels, where for our particular qubit-optomechanical scheme we will consider energy losses arising from each element.

As explained in a previous section, to achieve maximal qubit-cavity entanglement at time multiples of $2 \pi$, we need to attain moderate-to-strong optomechanical radiation pressure interaction. Within the single-photon strong or ultrastrong optomechanical coupling regime, photons and electronic states have been found to have been strongly dressed by phonon excitations of the mechanical mode. Therefore, when $g$ and $\lambda$ operate in this limit, one needs to consider that the singlephoton optomechanical coupling modifies the eigenstates of the system [41]. The corresponding master equation in the optomechanical dressed picture (DME), together with the dressed Lindbladian for the qubit element, is

$$
\begin{aligned}
\frac{d}{d t} \hat{\rho}(t)= & -i[\hat{H}, \hat{\rho}(t)]+\gamma_{m}\left(n_{\mathrm{th}}+1\right) \mathcal{L}\left[\hat{b}-g \hat{a}^{\dagger} \hat{a}\right] \hat{\rho}(t) \\
& +\kappa \mathcal{L}[\hat{a}] \hat{\rho}(t)+\gamma_{m} n_{\mathrm{th}} \mathcal{L}\left[\hat{b}^{\dagger}-g \hat{a}^{\dagger} \hat{a}\right] \hat{\rho}(t) \\
& +\Gamma\left(1+n_{q}\right) \mathcal{L}\left[\hat{\sigma}^{-}\right] \hat{\rho}(t)+\Gamma n_{q} \mathcal{L}\left[\hat{\sigma}^{+}\right] \hat{\rho}(t) \\
& +\frac{\gamma_{\phi}}{2} \mathcal{L}\left[\hat{\sigma}_{z}\right] \hat{\rho}(t)+\frac{4 \gamma_{m} g^{2}}{\ln \left(\frac{1+n_{\mathrm{th}}}{n_{\mathrm{th}}}\right)} \mathcal{L}\left[\hat{a}^{\dagger} \hat{a}\right] \hat{\rho}(t),
\end{aligned}
$$

where $\hat{H}$ corresponds to the Hamiltonian in Eq. (1), and the Lindblad superoperator term

$$
\mathcal{L}[\hat{O}] \hat{\rho}=2 \hat{O} \hat{\rho} \hat{O}^{\dagger}-\hat{\rho} \hat{O}^{\dagger} \hat{O}-\hat{O}^{\dagger} \hat{O} \hat{\rho}
$$

takes into account the dissipative mechanisms to a thermal reservoir with occupation number $n_{\text {th }} ; \kappa\left(\gamma_{m}\right)$ is the scaled (by the mechanical frequency) photon (phonon) decay. Notice that as we are operating in optical frequencies we have neglected its corresponding occupation number $n_{c} \ll 1$, i.e., only downwards transitions will take place $\kappa \mathcal{L}[\hat{a}] \hat{\rho}(t)$ in the optical energy ladder. We consider qubit decoherence channels described by the qubit relaxation $\Gamma$ and the dressed qubit dephasing $\gamma_{\phi}=\Gamma_{\phi}+4 \gamma_{m} \lambda^{2} / \ln \left[\frac{1+n_{\text {th }}}{n_{\text {th }}}\right]$, where $\Gamma_{\phi}$ is the qubit pure dephasing rate. Finally, we assume a common thermal reservoir for the composite system, i.e., each element of our qubit-optomechanical setup is in contact with the same environment at the same temperature; thus as $\lambda \sim 1$, we consider that $n_{q}=n_{\text {th }}$.

The DME found in Ref. [41] stands as a more general case when compared to the standard master equation (SME). One can transition between DME towards SME by simply considering $g \ll 1$, and consequently, the joint optomechanical decoherence channel $\mathcal{L}\left[\hat{b}-g \hat{a}^{\dagger} \hat{a}\right]$ can be effectively approximated to $\mathcal{L}[\hat{b}]$.

In current optomechanical systems, the primary decoherence channel is related to photon leakage from the cavity. Meanwhile, oscillator energy losses can be second-placed in the decoherence hierarchy. For this reason, we consider a mechanical oscillator with high mechanical quality factor $Q=10^{5}$ (according to our scaled definitions this translates into $\gamma=10^{-5}$ ). On the other hand, we will fix the photon
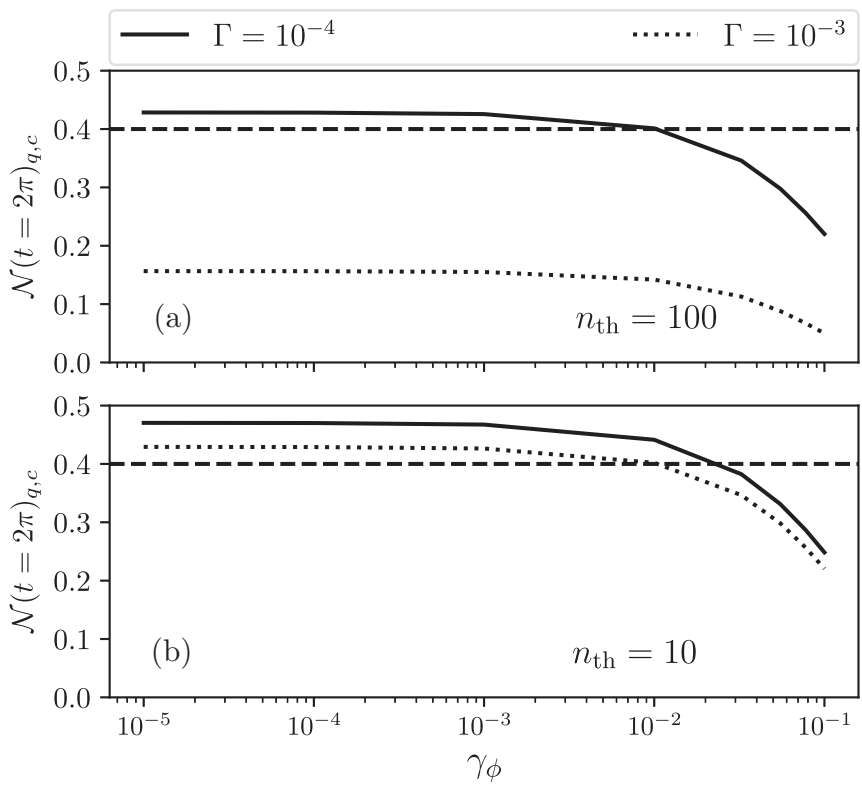

FIG. 5. Qubit-cavity entanglement at $t=2 \pi$ for different spin relaxation rates $\Gamma$ as a function of the spin dephasing rate $\gamma_{\phi}$. We fixed $\gamma_{m}=10^{-5}, \kappa=10^{-2}$, and other values as in Fig. 3 .

decay rate equal to $\kappa=0.01$ [42]. We vary the rest of the parameters in Fig. 5, for which we intend to illustrate up to which values of $\left\{\gamma_{\phi}, \Gamma\right\}$ the negativity at $t=2 \pi$ can be accommodated.

The particular case considered by us is the lossless scenario depicted in Fig. 3, where both the optics and the mechanics are initially in coherent states. In Fig. 5, we arbitrarily set a $20 \%$ attenuation of the maximal value of the entanglement achieved in the lossless case as the acceptable detrimental tolerance (negativity up to 0.4 in the black dashed line). Notice that although no cooling of the mechanical oscillator is truly obliged for our scheme to work, the thermal reservoir impacts on the qubit coherence. The parametric Hamiltonian, due to its qubit and photonic dispersive nature, demands a qubit coherence to reach nonzero entanglement between these parties. In other words, no fully mixed state or a $\hat{\sigma}_{z}$ eigenstate will entangle dynamically with the light field. Under the assumption of a common reservoir, $n_{\text {th }}=100\left[n_{\text {th }}=10\right]$ will require dephasing rates of $10^{-2}$ and qubit relaxing rates as low as $\Gamma=10^{-4}\left[\Gamma=10^{-3}\right]$.

\section{OPTICAL NONCLASSICAL STATES GENERATION}

We present how nonclassical states of the light field can be generated due to their dynamics alone and by performing measurements on the qubit state. The purpose of this section intends to be primarily illustrative, and thus we believe that some other optical nonclassical states can be attained and not readily covered in the following.

Notice that, in optomechanics it is already known that measurements on the mechanical object can conditionally project the cavity field into nonclassical states [26]. However, in practice, a qubit (being a digital measurement of 0 or 1) may be measured much more faithfully in comparison to a continuous position degree of freedom. Thus it is relevant to 
find whether nonclassical states of a cavity field can be prepared even when a qubit and the field are interfaced indirectly through the mechanical element. Here we show that this is indeed the case.

We distinguish two cases, namely, (i) the generation of the nonclassical state by evolution alone and (ii) by measuring the qubit subsystem. We obtain the first case from the reduced density matrix of the cavity field [see Eq. (13)]. At the particular time of $2 l \pi$ ( $l$ being an integer), the corresponding mixed state reads

$$
\begin{aligned}
\hat{\rho}_{c}(t=2 l \pi)= & e^{-|\alpha|^{2}} \sum_{n, m=0}^{\infty} \frac{\alpha^{n+m}}{\sqrt{n ! m !}} e^{2 i g^{2} l \pi\left(n^{2}-m^{2}\right)} \\
& \times \cos [4 \pi g l \lambda(m-n)]|n\rangle\langle m| .
\end{aligned}
$$

For a particular set of values of the scaled coupling parameters $g$ and $\lambda$, we can generate nonclassical states for the cavity mode, the so-called multicomponent Schrödinger cat states. The appropriate choice of $\{g, \lambda\}$ (when each party becomes disentangled from the rest) can be obtained from the intrinsic entanglement expression derived previously in Eq. (14), being $4 g \lambda l=k$ ( $\{k, l\}$ integers). The multicomponent Schrödinger cat states are achieved for $g \sqrt{2 l p}=1$, where $p \geqslant 2$ gives the $p$-mode Schrödinger cat state generated. Without loss of generality, let us fix $\{l, k\}=1$, as different values of those will involve only rotations in the phase space of the light field. In Figs. 6(a) and 6(b) we show the Wigner quasiprobability distributions [defined as $\left.W(x, y)=\int_{-\infty}^{\infty}\left\langle x+x^{\prime}\left|\hat{\rho}_{c}\right| x-x^{\prime}\right\rangle e^{-2 i y x^{\prime} / \hbar} d x^{\prime}\right]$ for the preparation of two- and five-component Schrödinger cat states, respectively. Notice that if we would like to dynamically generate the lower $p$-component Schrödinger cat states $(p=2)$, the qubit-optomechanical couplings are as strong as $g=\lambda=0.5$. In this sense, the strong-to-moderate single-photon coupling strength $g \sim 0.5$ might undermine the nonclassical optical production, as it remains challenging from an experimental point of view.

Furthermore, as $2 p l g^{2}=1$, it implies that $g$ is lower bounded by $g \geqslant 1 / \sqrt{4 l}$, and thus reducing $g$ to $10^{-2}$ (a more experimentally available optomechanical strength) will necessarily entail letting the system evolve for $l \sim 10^{3}$ oscillator's round trips; however, intracavity light photons for such $l$ may have leaked from the cavity at that time. One way to overcome this obstacle is to steer the light field into a nonclassical state, i.e., by projecting the qubit such that we can generate a twocomponent Schrödinger cat in the weak single-photon regime. To observe this mechanism, let us write the normalized optical field projected on $\sqrt{2}|+\rangle=|\uparrow\rangle+|\downarrow\rangle$ as follows:

$$
\begin{aligned}
\hat{\rho}_{c}(t=2 l \pi)= & \frac{1}{\mathcal{P}} \sum_{n, m=0}^{\infty} \frac{\alpha^{n+m}}{\sqrt{n ! m !}} e^{2 i g^{2} l \pi\left(n^{2}-m^{2}\right)} \\
& \times \cos (4 \pi g l \lambda n) \cos (4 \pi g l \lambda m)|n\rangle\langle m|,
\end{aligned}
$$

with normalization $\mathcal{P}=\sum_{n=0}^{\infty} \frac{\alpha^{2 n}}{n !} \cos ^{2}(4 \pi g l \lambda n)$. Moreover, we require the conditions found above to be relaxed for the case of $p$-component cat-state generation, as the weak regime $g \sim 10^{-2}$ is not considered in the above description. For the conditioned density matrix above, we can see that the cosine's angle should stand for $4 \pi g l \lambda=\pi / 2$ to generate a
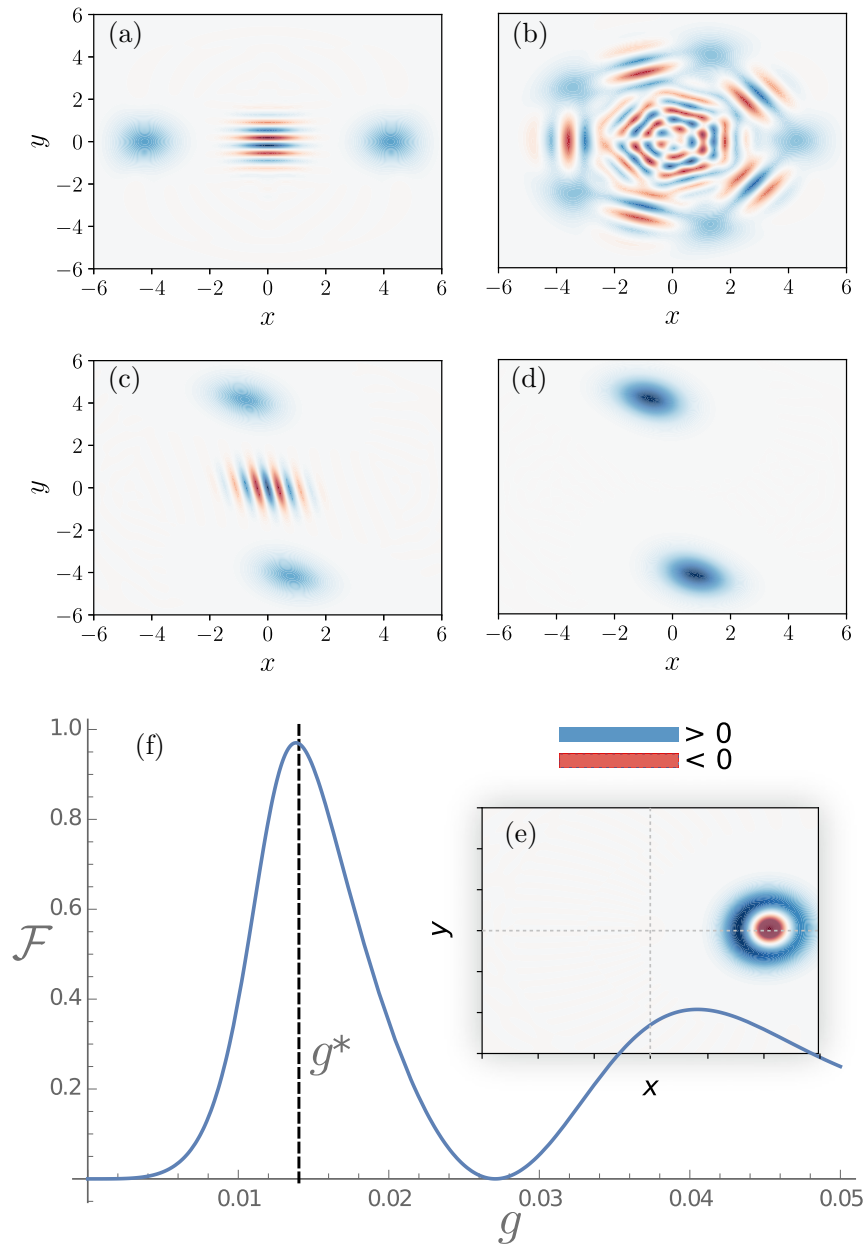

FIG. 6. Panels (a) and (b) show the Wigner distribution for the generation of nonclassical states for the light field due to its dynamics alone at $t=2 \pi$. In (a) we show the two-component cat state for $g=$ $\lambda=0.5$, while (b) corresponds to the five-component cat state for $g \sim 0.32$ and $\lambda \sim 0.8$; (c) illustrates the preparation of nonclassical states via qubit projection $|+\rangle$ at $t=10 \times 2 \pi$ when operating in the weak optomechanical regime $g \sim 0.013$ and qubit-mechanical coupling $\lambda=1$. In (d) we show the impossibility to obtain a nonclassical state with the same parameters used in (c) by its dynamics alone. In panel (e) we show the displaced Fock number state $\hat{D}(\alpha)|1\rangle$ via qubit projection with its corresponding fidelity shown in (f). Other values are $\alpha=3$, and $g^{*}$ the optimal optomechanical strenght to achieve $\hat{D}(\alpha)|1\rangle$. In all panels, positive (negatives) values in the Wigner function are blue (red) colored.

two-component cat state, i.e., $g=1 /(8 l \lambda)$. The proper rate between $\lambda$ and the oscillator's cycle can give rise to $g \sim 10^{-2}$. For instance, choosing $\lambda=1$ and $l=10$ makes $g \sim 0.012$, so we can readily obtain nonclassical states for the optics within this regime [see Fig. 6(c)]. It is also relevant to point out that no nonclassical states due to the tripartite dynamics alone were reported with $g \sim 0.012$ and $\lambda=1$ at times before $t=10 \times 2 \pi$ [see Fig. 6(d)]; only by measuring the qubit can one collapse the optical field into a nonclassical state [see Fig. 6(c)].

The hybrid quantum system, when $g \ll \lambda$, makes the typical nonlinear Kerr-like dynamics to evolve very slowly. In other words, the initial coherent amplitude of $\alpha$ 
requires several mechanical oscillations to exhibit nonclassical fringes in the Wigner function. This factor is relevant, as the Schrödinger cat state shown in Fig. 6(c) suggests that in earlier times, the coherent state for the optical field should have been located closer in the phase space; therefore we can generate a displaced "kitten" state (Schrödinger cat state with low amplitude) of the optical mode. To quantify this, we compare the actual displaced Fock number state defined in Fock basis as

$$
\begin{aligned}
\hat{D}(\alpha)|n\rangle= & e^{-\frac{\alpha^{2}}{2}} \sum_{r=0}^{\infty} \frac{\alpha^{r}}{r !} \sum_{j=0}^{n} \frac{(-\alpha)^{j}}{j !} \sqrt{\frac{(n-j+r) ! n !}{(n-j) !(n-j) !}} \\
& \times|n-j+r\rangle .
\end{aligned}
$$

\section{CONCLUDING REMARKS}

We show how parametric coupling can induce maximal qubit-cavity entanglement in an oscillator-mediated qubit-optomechanical system. Because of the dispersive offresonant qubit and photonic interaction, it is not readily evident that the qubit cavity will correlate maximally at some specific time. Here we demonstrate that the maximum value of the indirect qubit-cavity entanglement is a consequence of the oscillator disentanglement from the rest of the parties at each cycle. In addition to this, we show that the maximal value of the qubit-cavity negativity at the optimal time is independent of the phonon occupancy number for mechanical thermal mixtures, thus proving that the oscillator mediates between the qubit and the optics, with no requirement for cooling the mechanical oscillator to its ground state.

With use of the intrinsic entanglement $\mathcal{E}(t)$, we show that to attain such a maximum value one requires the system to be evolved into the moderate-to-strong single-photon optomechanical regime, while the qubit frequencies are comparable to the mechanical oscillator frequency $\lambda \sim 1$. Because of this operational regime, we solved a general master equation considering an optomechanical dressed picture. For feasible damping rates of the oscillator with quality factors up to $Q \sim 10^{5}$, and photon leaking rates of $\kappa \sim 10^{-2}$, we computed that the qubit relaxation decay $\Gamma$ and qubit dephasing rate $\gamma_{\phi}$ can be accommodated up to $\left\{\Gamma, \gamma_{\phi}\right\} \sim$ $\left\{10^{-3}, 10^{-2}\right\}$ when in contact with a thermal reservoir of $n_{\text {th }} \sim 10$ (increasing $n_{\text {th }} \sim 10^{2}$ requires to reduce $\Gamma 1$ order of magnitude).

Finally, we also illustrate how the generation of nonclassical states for the cavity field can be accomplished via evolution alone or by collapsing the cavity field through a local measurement on the qubit state. Such a technique can be more advantageous when compared to a purely optomechanical system, as in this system we need to measure the continuous degree of freedom of the mechanical oscillator [26]. Our proposal, a potential integrated hybrid node in the absence of the linearized optomechanical regime (see Ref. [43] for a method to entangle distant qubits using optomechanical transducers in the linearized optomechanical regime) and external driving, may open up the scope for quantum networking schemes even when the interactions are not of the energy exchange type.

\section{ACKNOWLEDGMENTS}

V.M. and G.D.M.N. acknowledge funding from the Chinese Postdoctoral Science Fund, Grants No. 2018M643435 and No. 2018M643436, respectively. S.B. would like to acknowledge EPSRC Grants No. EP/N031105/1 and No. EP/S000267/1.
[1] G. Kurizki, P. Bertet, Y. Kubo, K. Mølmer, D. Petrosyand, P. Rabl, and J. Schmiedmayer, Proc. Natl. Acad. Sci. USA 112, 3866 (2015).

[2] B. Rogers, N. Lo Gullo, G. De Chiara, G. M. Palma, and M. Paternostro, Quantum Meas. Quantum Metrol. 2, 11 (2014).

[3] M. Wallquist, K. Hammerer, P. Rabl, M. Lukin, and P. Zoller, Phys. Scr. T137, 014001 (2009).

[4] M. Wallquist, K. Hammerer, P. Zoller, C. Genes, M. Ludwig, F. Marquardt, P. Treutlein, J. Ye, and H. J. Kimble, Phys. Rev. A 81, 023816 (2010).

[5] S. J. M. Habraken, K. Stannigel, M. D. Lukin, P. Zoller, and P. Rabl, New J. Phys. 14, 115004 (2012)

[6] H. J. Kimble, Nature (London) 453, 1023 (2008).

[7] J. Restrepo, C. Ciuti, and I. Favero, Phys. Rev. Lett. 112, 013601 (2014); J. Restrepo, I. Favero, and C. Ciuti, Phys. Rev. A 95, 023832 (2017).

[8] J. I. Cirac, P. Zoller, H. J. Kimble, and H. Mabuchi, Phys. Rev. Lett. 78, 3221 (1997).

[9] T. Pellizzari, Phys. Rev. Lett. 79, 5242 (1997).

[10] S. J. van Enk, H. J. Kimble, J. I. Cirac, and P. Zoller, Phys. Rev. A 59, 2659 (1999).
[11] S. Bose, P. L. Knight, M. B. Plenio, and V. Vedral, Phys. Rev. Lett. 83, 5158 (1999).

[12] J. J. Pla, K. Y. Tan, J. P. Dehollain, W. H. Lim, J. J. L. Morton, D. N. Jamieson, A. S. Dzurak, and A. Morello, Nature (London) 489, 541 (2012).

[13] M. Maragkou, Nat. Mater. 14, 468 (2015).

[14] S. Kolkowitz, A. C. Bleszynski Jayich, Q. P. Unterreithmeier, S. D. Bennett, P. Rabl, J. G. E. Harris, and M. D. Lukin, Science 335, 1603 (2012).

[15] P. Rabl, P. Cappellaro, M. V. Gurudev Dutt, L. Jiang, J. R. Maze, and M. D. Lukin, Phys. Rev. B 79, 041302(R) (2009).

[16] P. Rabl, S. J. Kolkowitz, F. H. L. Koppens, J. G. E. Harris, P. Zoller, and M. D. Lukin, Nat. Phys. 6, 602 (2010).

[17] K. Stannigel, P. Rabl, A. S. Sørensen, P. Zoller, and M. D. Lukin, Phys. Rev. Lett. 105, 220501 (2010).

[18] K. Stannigel, P. Rabl, A. S. Sørensen, M. D. Lukin, and P. Zoller, Phys. Rev. A 84, 042341 (2011).

[19] K. Stannigel, P. Rabl, and P. Zoller, New J. Phys. 14, 063014 (2012).

[20] M. Scala, M. S. Kim, G. W. Morley, P. F. Barker, and S. Bose, Phys. Rev. Lett. 111, 180403 (2013). 
[21] A. D. Armour, M. P. Blencowe, and K. C. Schwab, Phys. Rev. Lett. 88, 148301 (2002).

[22] M. Abdi, M. Pernpeintner, R. Gross, H. Huebl, and M. J. Hartmann, Phys. Rev. Lett. 114, 173602 (2015).

[23] A. Olaya-Castro, N. F. Johnson, and L. Quiroga, J. Opt. B: Quantum Semiclassical Opt. 6, S730 (2004).

[24] J. R. Johansson, P. D. Nation, and F. Nori, Comput. Phys. Commun. 184, 1234 (2013); 183, 1760 (2012).

[25] V. Montenegro, A. Ferraro, and S. Bose, Phys. Rev. A 90, 013829 (2014).

[26] S. Bose, K. Jacobs, and P. L. Knight, Phys. Rev. A 56, 4175 (1997).

[27] S. Mancini, V. I. Man'ko, and P. Tombesi, Phys. Rev. A 55, 3042 (1997).

[28] S. Bose, K. Jacobs, and P. L. Knight, Phys. Rev. A 59, 3204 (1999).

[29] A. S. Parkins, P. Marte, P. Zoller, and H. J. Kimble, Phys. Rev. Lett. 71, 3095 (1993).

[30] K. Vogel, V. M. Akulin, and W. P. Schleich, Phys. Rev. Lett. 71, 1816 (1993).

[31] C. K. Law and H. J. Kimble, J. Mod. Opt. 44, 2067 (1997).

[32] S. P. Premaratne, F. C. Wellstood, and B. S. Palmer, Nat. Commun. 8, 14148 (2017).

[33] G. Vidal and R. F. Werner, Phys. Rev. A 65, 032314 (2002).

[34] M. R. Vanner, I. Pikovski, G. D. Cole, M. S. Kim, Č. Brukner, K. Hammerer, G. J. Milburn, and M. Aspelmeyer, Proc. Natl. Acad. Sci. USA 108, 16182 (2011).
[35] A. H. Safavi-Naeini, T. P. Mayer Alegre, J. Chan, M. Eichenfield, M. Winger, Q. Lin, J. T. Hill, D. E. Chang, and O. Painter, Nature (London) 472, 69 (2011).

[36] J. D. Teufel, T. Donner, D. Li, J. H. Harlow, M. S. Allman, K. Cicak, A. Sirois, J. D. Whittaker, K. W. Lehnert, and R. W. Simmonds, Nature (London) 475, 359 (2011).

[37] K. W. Murch, K. L. Moore, S. Gupta, and D. M. Stamper-Kurn, Nat. Phys. 4, 561 (2008).

[38] A. Xuereb, C. Genes, and A. Dantan, Phys. Rev. Lett. 109, 223601 (2012).

[39] J. Chan, T. P. Mayer Alegre, A. H. Safavi-Naeini, J. T. Hill, A. Krause, S. Gröblacher, M. Aspelmeyer, and O. Painter, Nature (London) 478, 89 (2011).

[40] A. D. O'Connell, M. Hofheinz, M. Ansmann, Radoslaw C. Bialczak, M. Lenander, Erik Lucero, M. Neeley, D. Sank, H. Wang, M. Weides, J. Wenner, John M. Martinis, and A. N. Cleland, Nature (London) 464, 697 (2010); V. Montenegro, R. Coto, V. Eremeev, and M. Orszag, Phys. Rev. A 98, 053837 (2018); D. D. Bhaktavatsala Rao, S. A. Momenzadeh, and J. Wrachtrup, Phys. Rev. Lett. 117, 077203 (2016).

[41] D. Hu, S.-Y. Huang, J.-Q. Liao, L. Tian, and H.-S. Goan, Phys. Rev. A 91, 013812 (2015); R. Betzholz, J. M. Torres, and M. Bienert, ibid. 90, 063818 (2014).

[42] M. Aspelmeyer, T. J. Kippenberg, and F. Marquardt, Rev. Mod. Phys. 86, 1391 (2014).

[43] O. Černotík and K. Hammerer, Phys. Rev. A 94, 012340 (2016). 